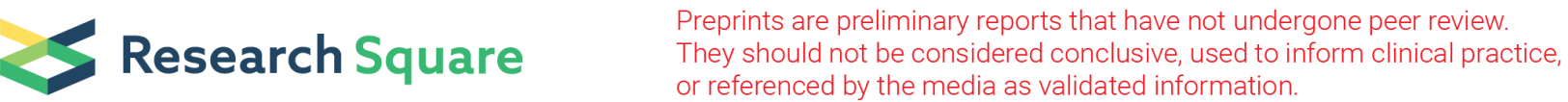

\section{Metformin Exerts An Antitumor Effect By Inhibiting Bladder Cancer Cell Migration And Growth, And Promoting Apoptosis Through The PI3K/AKT/mTOR Pathway}

\section{Zhiyong Shen}

The Third Affiliated Hospital of Soochow University

\section{Dong Xue}

The Third Affiliated Hospital of Soochow University

\section{Kun Wang}

The Third Affiliated Hospital of Soochow University

\section{Facai Zhang}

Sichuan University

Jiaqi Shi

The Affiliated Hospital of Guizhou Medical University

\section{Benzhong Jia}

The Affiliated Cancer Hospital of Guizhou Medical University

\section{Dan Yang}

The Affiliated Hospital of Guizhou Medical University

\section{Qianjin Zhang}

The Third Affiliated Hospital of Soochow University

\section{Shuai Zhang}

Laboratory of the Affiliated Cancer Hospital of Guizhou Medical University

\section{Hongyu Jiang}

Laboratory of the Affiliated Cancer Hospital of Guizhou Medical University

\section{Daiqin Luo}

The Affiliated Hospital of Guizhou Medical University

\section{Xueying Li}

Laboratory of the Affiliated Cancer Hospital of Guizhou Medical University

\section{Quliang Zhong}

The Affiliated Hospital of Guizhou Medical University

Junhao Zhang

The Affiliated Hospital of Guizhou Medical University

\section{Zheng Peng}

The Affiliated Cancer Hospital of Guizhou Medical University 


\section{Yu Han}

The Affiliated Cancer Hospital of Guizhou Medical University

\section{Chongyang Sima}

The Affiliated Cancer Hospital of Guizhou Medical University

\section{Xiaozhou He}

The Third Affiliated Hospital of Soochow University

Lin Hao ( $D$ haolinxuzhou@163.com)

Xuzhou Central Hospital

\section{Research Article}

Keywords: Bladder cancer T24 cells, Bladder cancer 5637 cells, Metformin, PI3K/AKT/mTOR signaling pathway

Posted Date: September 24th, 2021

DOl: https://doi.org/10.21203/rs.3.rs-846120/v1

License: (a) (i) This work is licensed under a Creative Commons Attribution 4.0 International License. Read Full License 


\section{Abstract}

Background To observe and explore the effect of metformin on the migration,and proliferation of bladder cancer T24 and 5637 cells in vitro.

Methods Bladder cancer T24 and 5637 cell lines were cultured in vitro, and were divided into group A (blank control group) and group B (metformin group: 5, 10, 15, and $20 \mathrm{mmol} / \mathrm{L}$ ); both groups were plated on 6-well plates at the same time. Culture in 24-well plates was used for wound healing assays and in 96well plates for Transwell migration and invasion, and Cell Counting Kit-8 proliferation experiments. We observed and detected the cell migration and proliferation ability of each group at 48 hours, and calculated the cell migration area and survival rate. Flow cytometry was used to detect cell apoptosis in the groups. The apoptosis-related protein, cleaved-caspase 3, cleaved-PARP and PI3K/AKT/mTOR signaling pathway member proteins PI3K, phosphorylated (p)-PI3K, AKT, p-AKT, mTOR, and p-mTOR were detected using western blotting.

Results After 48 hours of treatment with different concentrations of metformin, the cell migration and proliferation capabilities were significantly lower than those in the blank control group. The proliferation and migration abilities of T24 and 5637 cells decreased in a metformin concentration-dependent manner $(P<0.05)$. The apoptosis rate under different concentrations of metformin, as detected by flow cytometry, showed a significantly higher rate in the metformin group than in the control group ( $P \otimes 0.05)$. Compared with that in the control group, the level of cleaved-caspase 3 and cleaved-parp protein in the metformin group was increased in each treatment group, and the levels of p-mTOR, p-AKT, and p-PI3K decreased significantly compared with those in the control group $(P<0.05)$.

Conclusion Metformin inhibit bladder cancer T24 and 5637 cell migration and proliferation, and induced their apoptosis. The mechanism might involve inhibition of the activation of the PI3K/AKT/mTOR signaling pathway.

\section{Background}

Bladder cancer is a common malignant tumor of the urinary system[1]. Clinically, approximately $70 \%$ of bladder cancer cases are non-muscular invasive bladder cancer (NMIBC) and $30 \%$ are muscularly invasive bladder cancer (MIBC)[2-3]. NMIBC is characterized by a high rate of recurrence and a low rate of mortality. For NMIBC, the preferred surgical treatment method is transurethral electric resection of bladder tumor (TURBT) and routine postoperative infusion of chemotherapy drugs to prevent tumor recurrence and progression; however, active postoperative adjuvant therapy still results in a high recurrence rate. Metformin is a clinical drug used routinely to treat type 2 diabetes. We reviewed the domestic and foreign literature and found that metformin can significantly reduce the incidence of pancreatic cancer, liver cancer, breast cancer, and other malignant tumors [3, 4], in addition to improving the prognosis of patients with type 2 diabetes $[5,6]$. These findings suggested that metformin had a potential antitumor effect. Therefore, from September 2019 to May 2021, we observed metformin's effects on bladder cancer 
T24 and 5637 cell proliferation and migration, and explored its possible molecular mechanisms. We aimed to provide a theoretical basis for the use of metformin to treat bladder cancer.

\section{Methods}

\subsection{Reagents and Cell culture}

Reagents: Human bladder cancer cell lines T24 and 5637 were provided by the cell bank of the Chinese Academy of Sciences in Shanghai. The present study used metformin (Shanghai Beyotime Biotechnology Co., Ltd., Shanghai, China), Roswell Park Memorial Institute (RPMI)-1640 medium (Cellmax Company, Groningen, Netherlands), fetal bovine serum (FBS) (Sigma, St. Louis, MO, USA), phosphatebuffered saline (PBS) (Beijing Solarbio Company, Beijing, China), Cell counting Kit-8 (CCK-8) (Shanghai Beyotime Biotechnology Co., Ltd., Shanghai, China), and an Annexin V-fluorescein isothiocyanate (FITC)/propidium iodide (PI) Apoptosis Assay Kit (BD Biosciences, San Jose, CA, USA). Antibodies recognizing $\mathrm{PI} 3 \mathrm{~K} / \mathrm{Akt} / \mathrm{mTOR}$ signaling pathway associated proteins (phosphatidylinositol-4,5bisphosphate 3-kinase (PI3K), phosphorylated (p)-PI3K, protein kinase B (Akt), p-Akt, mechanistic target of rapamycin (mTOR), p-mTOR), glyceraldehyde-3-phosphate dehydrogenase (GAPDH), and Apoptosisrelated protein (cleaved-caspase 3 and cleaved-poly(ADP-ribose) polymerase (PARP)) were all purchased from Affinity Biosciences (Cincinnati, OH, USA). We also used a microplate analyzer (Thermo Scientific, Waltham, MA, USA), a flow cytometer (Bio-Rad, Hercules, CA, USA), and a western blotting imaging system (Bio-Rad).

Cell culture: Bladder cancer T24 and 5637 cells were taken out of $-80^{\circ} \mathrm{C}$ liquid nitrogen storage and thawed in a beaker for $1 \mathrm{~min}$. The cells were suspended in RPMI-1640 medium containing $10 \% \mathrm{FBS}$ and placed into an incubator at $37{ }^{\circ} \mathrm{C}$ and $5 \% \mathrm{CO}_{2}$ for culture. After the cell density reached about $90 \%$, the cells were passaged. T24 and 5637 cells in the logarithmic growth phase were used for the following experiments.

\subsection{Wound healing assay}

We made horizontal lines on the back of the 6-well plate, about every $0.5-1 \mathrm{~cm}$, across the holes. A single cell suspension was prepared by adding $0.25 \%$ trypsin to the cells for digestion. The cell plate count method was used to adjust the cell concentration to about $5.0 \times 10^{5} / \mathrm{mL}$ and the cells were inoculated into a 6-well plate. The control group (A) and Group B (metformin 5, 10, 15, and $20 \mathrm{mmol} / \mathrm{L}$ ) were placed in an incubator for $24 \mathrm{~h}$ until the cells were attached to the wall and a confluent cell layer had developed. Scratches were made in the cell layer using a micropipette tip. The cells were washed with PBS three times to remove the scratched cells. Serum-free RPMI-1640 medium was added vertically and gently at 
the apex of the plate. Photographs were taken at time zero and after 48 hours of culture using an Olympus camera (Tokyo, Japan).

\subsection{Transwell assay}

A single cell suspension was prepared by adding $0.25 \%$ trypsin to the cells for digestion. $500 \mu \mathrm{L}$ of medium containing $10 \%$ FBS was added to the lower chamber of the Transwell apparatus ( $8 \mu \mathrm{m}$ in diameter), and $200 \mu \mathrm{L}$ of cell suspension without serum was added to the upper chamber. After incubation for $48 \mathrm{~h}$, the medium in the upper and lower chambers was removed, and $500 \mu \mathrm{L}$ of $4 \%$ paraformaldehyde was added to the lower chamber to fix the cells for half an hour, after which the paraformaldehyde was removed. The cells that did not penetrate the membrane were wiped off using a cotton swab. Then, $500 \mu \mathrm{L}$ of $0.1 \%$ crystal violet were added to the lower chamber to stain the cells for 15 minutes. The chamber was washed with PBS until it was completely clear, and the cells were observed and counted under a microscope (Olympus).

\subsection{Assay for cell growth}

A Cell Counting Kit-8 (CCK-8) assay was used to determine cell proliferation. Logarithmic growth phase cells were digested with $0.25 \%$ trypsin, and a single cell suspension was prepared. The cell density was adjusted to about $1 \times 10^{3} / \mathrm{mL}$, and $100 \mu \mathrm{L}$ cells of the suspension were inoculated in each well of a 96-well plate, and cultured in an incubator for $24 \mathrm{~h}$. After the cells adhered to the wall, the medium was discarded and $5,10,15$, and 20 mmol $\backslash L$ metformin were added and incubated for $48 \mathrm{~h}$, respectively. Five replicates were set for each concentration, and a blank control group was set with the same volume of medium. After the treatment, each well received $10 \mu \mathrm{L}$ of CCK-8 solution, the 96 -well plate was gently knocked to mix, and then incubated for 1.5 hours. The absorbance value at $450 \mathrm{~nm}$ was measured using an enzyme plate analyzer (Thermo Scientific)

\subsection{Assay for colony formation}

The proliferation of T24 and 5637 cells was determined using a colony formation assay. T24 and 5637 cells were plated in 6-well plates at a density of 100 cells per well and cultured for $12 \mathrm{~h}$. Thereafter, the cells were treated with metformin at $5,10,15$, and $20 \mathrm{mmol} / \mathrm{L}$, or with medium only (RPMI-1640 medium $+10 \%$ FBS). The culture medium was changed every three days and observed continuously for at least 10-12 days. Thereafter, $4 \%$ paraformaldehyde was used to fix the cells, followed by staining with using $0.1 \%$ Crystal violet solution. The stained cells were counted under an inverted microscope (Olympus).

\subsection{Assay of cell apoptosis}


Cell apoptosis was detected using flow cytometry. Logarithmic growth phase cells were digested with $0.25 \%$ trypsin, and a single cell suspension was prepared. The density of the cells was adjusted to $1.0 \times$ $10^{5} / \mathrm{mL}$, and the cells were inoculated in a 6-well plate at $1 \mathrm{~mL} / \mathrm{well}$. The cells were cultured in an incubator for $24 \mathrm{~h}$. After cell adherence, we discarded the medium and the cells were divided into the control group and the metformin group, which was treated with 5, 10, 15, and $20 \mathrm{mmol} / \mathrm{L}$ metformin for $48 \mathrm{~h}$, respectively, and the control group was treated with the same volume of medium. Cells in each group were collected and rinsed with precooled PBS three times. After centrifugation at $1500 \times g$ for 5

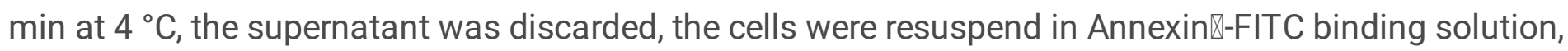
mixed with PI staining solution, and incubated in the dark for $15 \mathrm{~min}$. The apoptosis rate was detected by flow cytometry, carried out according to the manufacturers' protocol (Cell Sorter BD FACSAria II, BD Biosciences)

\subsection{Western blotting analysis}

Logarithmic phase cells were and digested using $0.25 \%$ trypsin to prepare a single cell suspension, and the cell density was adjusted to $1.0 \times 10^{6} / \mathrm{mL}$. The cells were inoculated in a 6 -well plate at $1 \mathrm{~mL} /$ well, and cultured in an incubator for $24 \mathrm{~h}$. After the cells had attached to the wall, we discarded the medium and the cells were divided into control group and metformin group. The metformin group was treated with $5,10,15$, and $20 \mathrm{mmol} / \mathrm{L}$ metformin for $48 \mathrm{~h}$, and the control group was treated with the same volume of medium containing $10 \%$ FBS. The cells were collected, and cell lysis buffer containing protease and phosphatase inhibitors was added and the cells were lysed on ice for $20 \mathrm{~min}$. After centrifugation at 1500 $\times g$ for 5 min at $4{ }^{\circ} \mathrm{C}$, the supernatant was retained and its protein concentration was determined using the bicinchoninic acid (BCA) method. Then, $40 \mathrm{mg}$ of protein was subjected to SDS-polyacrylamide gel electrophoresis, and the separated proteins were transferred to $0.45 \mu \mathrm{m}$ or $0.22 \mathrm{~mm}$ polyvinylidene fluoride (PVDF) membranes, and blocked using 5\% skimmed milk at room temperature for $40 \mathrm{~min}$. Primary antibodies were added (the dilution ratio of cleaved-caspase 3 and cleaved-PARP was 1:500, the dilution ratio of PI3K, p-PI3K, Akt, p-Akt, mTOR and p-mTOR was 1:1000, and the dilution ratio of GAPDH was $1: 10,000$ ), and placed in a refrigerator at $4{ }^{\circ} \mathrm{C}$ for overnight incubation. The membranes were washed with 1' Tris-buffered saline-Tween 20 (TBST) three times for 5 min each time and incubated with labeled secondary antibody at room temperature for $40 \mathrm{~min}$. After washing with $1 \times$ TBST three times $(5 \mathrm{~min}$ each time), ECL exposure luminescence solution was added at a 1:1 ratio onto the PVDF membrane for luminescence development. The immunoreactive protein bands were analyzed using Image-J software. The relative level of each protein was represented by the ratio of the gray level of target protein band to the gray level of internal reference, GAPDH. For the phosphorylated proteins, the relative expression level was represented by the ratio of the gray level of the phosphorylated target protein to that of the total target protein. Finally, the ChemiDocxRS imaging system was used to obtain images of the protein bands, which were analyzed using Quantity One software (Bio-Rad).

\subsection{Statistical analysis}


SPSS 22.0 statistical software (IBM Corp., Armonk, NY, USA) was used for the statistical analyses. Measurement data were expressed as the mean $\pm \mathrm{SD}$. One-way analysis of variance was used for comparisons between multiple groups. GraphPad Prism 8.0 (GraphPad inc., La Jolla, CA, USA) was used to draw the graphs and Image-J software ( $\mathrm{NIH}$, Bethesda, MD, USA) was used to calculate the western blotting gray values, cell counts and wound areas. Differences were judged to be statistically significant at a $P$ value $<0.05$.

\section{Results}

\subsection{Different concentrations of metformin inhibited the migration of T24 and 5637 cells in vitro}

Metformin at different concentration inhibited the migration of T24 and 5637 cells, as demonstrated by wound healing assay and Transwell chamber assays (Fig. 1A,B). The migration ability of T24 and 5637 cells decreased with increasing metformin concentration and the higher concentration of metformin inhibited the migration of T24 and 5637 significantly compared with that of the control $(P<.05)$.

\subsection{T24 and T5637 cell proliferation was inhibited by different concentrations of metformin in vitro}

The effects of different concentrations of metformin on T24 and 5637 cell proliferation were tested using CCK-8 assays (Fig. 2A,B) and colony formation assays (Fig. 2C). The proliferative ability of T24 and 5637 cells was inhibited by increasing concnetrations of metformin compared with the control $(P<0.05)$.

\subsection{Different concentrations of metformin promoted apoptosis of T24 and T5637 cells in vitro}

Metformin increased the rate of apoptosis in a concentration-dependent manner compared with that of the control $(P<0.05$; Figure 3$)$. The highest concentration of metformin significantly promoted cell apoptosis compared with that of the control $(P<0.05)$. The levels of cleaved-caspase- 3 and cleavedPARP increased significantly in T24 and 5637 cells treated with highest concentration of metformin $(P<$ 0.05). Interestingly, the lowest concentration of metformin did not increase the level of cleaved-caspase 3 and cleaved-PARP $(P>0.05)$, but with the increase in metformin concentration, cleaved-caspase 3 and cleaved-PARP protein levels increased significantly $(P<0.05)$.

\subsection{The PI3K/AKT/mTOR pathway is inhibited by different concentrations of metformin}

PI3K, Akt, and mTOR phosphorylation in T24 and 5637 cells was significantly reduced by metformin in a concentration-dependent manner $(P<0.05$; Fig. 4). 


\section{Discussion}

In the urinary system, the most common malignancy is bladder cancer, ranking as the 4th most common malignancy in men and the 11th most common malignancy in women in the United States. The incidence and mortality of bladder cancer in males is approximately four times that of females [1]. In China in 2019, there were 549,393 cases of bladder cancer, with an initial incidence of 7.2 per 100,000, ranking as 10th among all tumors. There were almost the same number of new cases in China $(82,270)$ as there were in the in the USA $(82,501)$ [2]. Clinically, about $70 \%$ of bladder cancers are NMIBC and $30 \%$ are MIBC. NMIBC has a low mortality rate and a high recurrence rate, whereas about $50 \%$ of cases of MIBC are potentially fatal [3]. For patients with NMIBC, the preferred treatment is TURBT with conventional postoperative infusion chemotherapy using the Bacillus Calmette-Guérin (BCG) vaccine (BCG), epirubicin (EPI), pirubicin (THP), and other cytotoxic drugs to prevent postoperative tumor recurrence and progression [4]. Despite active postoperative adjuvant treatment, the $5 y e a r$ recurrence rate remains high $(50-70 \%)$ among patients [5-7].

Metformin safely reduces insulin resistance and lowers blood glucose [8-9]. It is commonly used in the clinic to treat type II diabetes mellitus and has become popular in tumor treatment research because of its extensive anti-tumor effects. Metformin has anti-tumor effects in a variety of tumors, such as breast cancer, colon cancer, stomach cancer, pancreatic cancer, prostate cancer, lung cancer, and liver cancer [10-15]. Our current understanding of the mechanism by which metformin inhibits tumor cell invasion, migration, and proliferation can be roughly divided into the following pathways [10-16]: 1. Metformin inhibits the downstream mTOR pathway by activating the AMP-activated protein kinase (AMPK) signaling pathway to inhibit tumors; 2 . Metformin inhibits tumors by activating reactive oxygen species (ROS) and the C-Jun N-Terminal Kinase 1 (JNK) signaling cascade; 3 . Metformin can enhance the cytotoxicity of chemotherapy drugs. However, there has been only one study on the molecular mechanism of metformin when used in bladder cancer [17]. Our results indicated that under $5,10,15$, and $20 \mathrm{mmol} / \mathrm{L}$ metformin treatment for $48 \mathrm{~h}, \mathrm{~T} 24$ and 5637 cell survival was lower compared with that of the control group. The migration ability of T24 and T5637 cells treated with metformin was significantly weakened in a concentrationdependent manner, as assessed using wound healing and Transwell chamber assays. Thus, metformin can inhibit the migration and proliferation abilities of T24 and T5637 cells, which suggested that it would inhibit bladder cancer progression.

Programmed cell death (PCD) comprises mainly apoptosis (type I) and autophagy (type II) (17). The most common type of PCD is apoptosis, which is under the control of both intracellular and extracellular signals. Apoptosis is characterized by changes in cell morphology, e.g., mitosis and DNA condensation, production of membranous vesicles, cell contraction, and the development of apoptotic bodies. In last 30 years, apoptotic signaling pathways have been intensively studied in many types of tumor cells. Tumor apoptosis-related protein include the caspase family and the B-cell CLL/lymphoma 2 (Bcl-2) family, that latter of which has vital functions mitochondriamediated apoptosis. The pro-apoptotic BCL2 associated $\mathrm{X}$ protein (Bax) promotes mitochondrial cytochrome $\mathrm{C}$ through regulating the permeability of the mitochondrial membrane and activating the caspase cascade reaction, which ultimately leads to cell 
apoptosis. In our apoptosis experiment, the level of the apoptosis promoter cleaved-caspase 3 was increased by the different concnetrations of metformin treatment groups at different concentrations. In addition, flow cytometry showed that a higher proportion of T24 and T5637 bladder cancer cells underwent apoptosis after treatment with different concentrations of metformin.

Tumorigenesis and development are complex processes and extensive research on the molecular mechanism of tumors has revealed that tumorigenesis and development are related to multiple signaling pathways, among which PI3K/Akt /mTOR is the canonical pathway. Domestic and foreign scholars have discovered that this signaling pathway plays a crucial role in the occurrence and development of breast cancer, cervical cancer, pancreatic cancer, and other tumors [18]. PI3K/Akt signaling is associated with cell autophagy, apoptosis, and proliferation, and inflammation. In addition, in this pathway, mTOR acts a downstream regulator, exerting a vital function in autophagy and protein synthesis [19-22]. Although there have been few studies about metformin in bladder cancer, analysis of reviews and published studies revealed that more than $40 \%$ of patients with urothelial carcinoma had a dysregulated $\mathrm{PI3K} / \mathrm{AKT} / \mathrm{mTOR}$ pathway [23-28]. These observations inspired us to consider whether metformin also has an inhibitory effect in bladder cancer and if this effect is related to the PI3K pathway. In our in vitro study, we found that metformin indeed inhibited the PI3K/Akt/mTOR signaling pathway, and different concentrations of metformin reduced the levels of phosphorylated PI3K, Akt, and mTOR.

In conclusion, metformin inhibits bladder cancer T24 and 5637 cell migration and proliferation, activates the caspase cascade signaling pathway, and induces cell apoptosis. The mechanism might be associated with inhibition of the activation of the PI3K/Akt/mTOR signaling pathway.

\section{Abbreviations}

BC: Bladder cancer; PI3K: phosphatidylinositol-4,5-bisphosphate 3-kinase; p-PI3K: phosphorylated (p)PI3K; Akt: protein kinase B; p-Akt: phosphorylated Akt; mTOR: mechanistic target of rapamycin; p-mTOR: phosphorylated mTOR), GAPDH: glyceraldehyde-3-phosphate dehydrogenase; PARO: poly(ADP-ribose) polymerase (PARP).

\section{Declarations}

Ethics approval and consent to participate

Not applicable

Consent for publication

Not applicable

Vailability of data and materials 
The datasets used and/or analyzed during the current study are available from the corresponding author or the first author on reasonable request.

\section{Competing interests}

The authors declare that there are no conflicts of interests.

\section{Funding}

This work was supported by the Health Research Project from the Guizhou Municipal Health Commission, China [grant number Gzwkj2021-214], and was funded by the Science and Technology Project of Guizhou Province, China [grant number Qian Ke He LH [2016]-7396]

\section{Authors' contributions}

(I) Conception and design: Z Shen, X He; (II) Administrative support: All authors; (III) Provision of study material or patients: S Zhang, Y Jiang; (IV) Collection and assembly of data: Y Li, C Sima; (V) Data analysis and interpretation: Z Shen, D Yang, J Zhang; (VI) Manuscript writing: Z Shen, F Zhang, Y Li; (VII) Final approval of manuscript: All authors.

\section{Acknowledgments}

We thanks all professors and teachers who helped us and participated in this study and those who donated materials.

\section{References}

1. Siegel RL, Miller KD, Jemal A. Cancer statistics, 2017. CA Cancer J Clin, 2017,67(1):7-30.

2. Guan Youyan, Xing Nianzeng, Shou Jianzhong. The epidemiological status of urinary malignancies in China [J].Cancer Research, 2019, 22 (1):67-72.

3. Marcos-gragera R, Mallone S, Kiemeney LA. Urinary tract cancer survival in Europe 1999-2007: results of the population-based study EUROCARE-5 [J]. Eur J Cancer, 2015, 51(15): 2217-30.

4. EAU European Association of Urology Guidelines Non-muscle-invasive Bladder Cancer (TaT1 and $\mathrm{CIS})(2019$ edition)[DB/OL].

5. Richard J, Maurizio A, Wim J. Long-term efficacy results of EORTC genito-urinary group randomized phase 3 study 30911 comparing intravesical instillations of epirubicin, bacillus Calmette-Guérin, and bacillus Calmette-Guérin plus isoniazid in patients with intermediate-and high-risk stage TaT1 urothelial carcinoma of the bladder [J]. Eur Urol. 2010 May;57(5):766-73. doi:

10.1016/j.eururo.2009.12.024. Epub 2009 Dec 18.

6. Xiu-Wu Pan Lin Li Yi Huang.Icaritin acts synergistically with epirubicin to suppress bladder cancer growth through inhibition of autophagy [J]. ONCOLOGY R 334 EPORTS 35: 334 - 42, 2016 
7. PAN XW, LI L, HUANG Y.Icaritin acts synergistically with epirubicin to suppress bladder cancer growth through inhibition of autophagy. Oncology Rep 2016; 35: 334-42.

8. Coyle C, Cafferty FH, Vale C, Langley RE. Metformin as an adjuvant treatment for cancer: a systematic review and meta-analysis. Annals of oncology: official journal of the European Society for Medical Oncology. 2016;27:2184-95.

9. Morris A. Diabetes: Systemic effects of metformin revealed. Nature reviews Endocrinology.2017;13:562.

10. Grace H, Meloja S. Association of Metformin with Breast Cancer Incidence and Mortality in Patients with Type II Diabetes: A GRADE-Assessed Systematic Review and Meta-analysis [J]. Cancer Epidemiol Biomarkers Prev. 2018 Jun;27(6):627-35. doi: 10.1158/1055-9965.EPI-17-0936. Epub 2018 Apr 4.

11. Zar C, Nurul H. Is Metformin a Therapeutic Paradigm for Colorectal Cancer: Insight into the Molecular Pathway [J]. Curr Drug Targets.2017;18(6):734-50. doi: 10.2174/1389450118666161205125548.

12. Sarah C.The therapeutic potential of metformin in gastric cancer [J]. Gastric Cancer. 2019 Jul;22(4):653-62. doi: 10.1007/s10120-019-00952-w. Epub 2019 Mar 21.

13. Guoxing W, Xue S, et al. Survival Benefit of Metformin Adjuvant Treatment For Pancreatic Cancer Patients: a Systematic Review and Meta-Analysis [J]. Cell Physiol Biochem.2018;49(3):837-47.doi: 10.1159/000493214. Epub 2018 Sep 5.

14. Jessica W, Claire M E, Prasanna S. Metformin and Prostate Cancer: a New Role for an Old Drug [J]. Curr Urol Rep. 2017 Jun;18(6):46.doi: 10.1007/s11934-017-0693-8.

15. S Zhong, $Y$ Wu, $X$ Yan. Metformin use and survival of lung cancer patients: Meta-analysis findings [J]. Indian J Cancer. Jan-Mar 2017;54(1):63-67.doi: 10.4103/0019-509X.219582.

16. Bo L, pingting Z, Kenhan X,Tianrui C,Jian J,Haifeng W,et al.Metformin induces cell cycle arrest, apoptosis and autophagy through ROS/JNK signaling pathway in human osteosarcoma [J]Biol Sci.2020;6(1): 74-84.

17. Amr A.New insight for metformin against bladder cancer[J].EL-Arabey Genes and Environment (2017) 39:13 DOI 10.1186/s41021-017-0074-z

18. Yang J, Pi C, Wang G. Inhibition of PI3K/Akt /mTOR pathway by apigenin induces apoptosis and autophagy in hepatocellular carcinoma cells [J]. Biomed Pharmacother, 2018, 103 (2): 699-07.

19. Chen $H$, Qu Y, Tang B, Xiong T, Mu D. Role of mammalian target of rapamycin in hypoxic or ischemic brain injury: potential neuroprotection and limitations. Rev Neurosci.2012;23:279-287.

20. Li T, Zhu D, Mou T. IL-37 induces autophagy in hepatocellular carcinoma cells by inhibiting the PI3K/AKT/mTOR pathway. Mol Immunol. 2017;87:132-40.

21. Wang $S$, Chen $Y$, Chen N. Hydrogen sulfide promotes autophagy of hepatocellular carcinoma cells through the PI3K/Akt/mTOR signaling pathway. Cell Death Dis. 2017;8:e2688.

22. Kim J, Jiang J, Badawi M, Schmittgen T. miR-221 regulates CD44 in hepatocellular carcinoma through the PI3K-AKT-mTOR pathway. Biochem Biophys Res Commun. 2017;487:709-715. 
23. Marina P, Guilhem R, Anne-Laure C. Safety and efficacy of temsirolimus as second line treatment for patients with recurrent bladder cancer. BMC Cancer (2018) 18:194.

24. Houédé N, Pourquier P. Targeting the genetic alterations of the PI3K-AKTmTOR pathway: its potential use in the treatment of bladder cancers. Pharmacol Ther. 2015;145:1-18

25. Fechner G, Classen K, Schmidt D, Hauser S, Müller S. Rapamycin inhibits in vitro growth and release of angiogenetic factors in human bladder cancer. Urology. 2009;73:665-69.

26. Schedel F, Pries R, Thode B, Wollmann B, Wulff S, Jocham D, et al. mTOR inhibitors show promising in vitro activity in bladder cancer and head and neck squamous cell carcinoma. Oncol Rep. 2011;25:763-68.

27. Mansure JJ, Nassim R, Chevalier S, Rocha J, Scarlata E, Kassouf W. Inhibition of mammalian target of rapamycin as a therapeutic strategy in the management of bladder cancer. Cancer Biol Ther. 2009;8:2339-47.

28. Vasconcelos-Nóbrega C, Pinto-Leite R, Arantes-Rodrigues R, Ferreira R, Brochado P, Cardoso ML, et al. In vivo and in vitro effects of RAD001 on bladder cancer. Urol Oncol.2013;31:1212-21.

\section{Figures}




\section{A)T24}

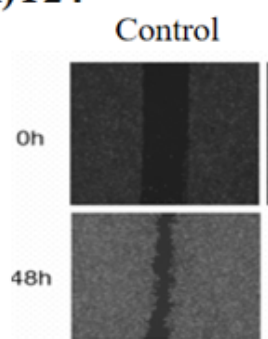

Control

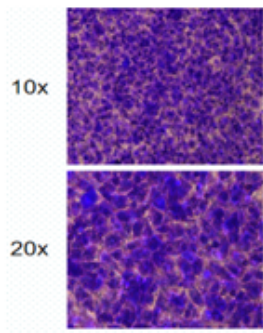

\begin{abstract}
$5 \mathrm{mM}$
\end{abstract}

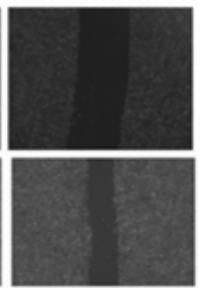

$5 \mathrm{mM}$

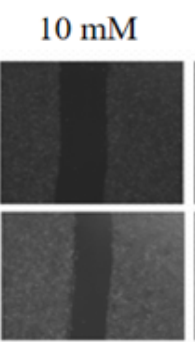

$10 \mathrm{mM}$

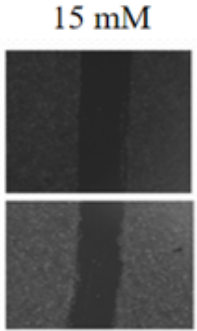

$15 \mathrm{mM}$

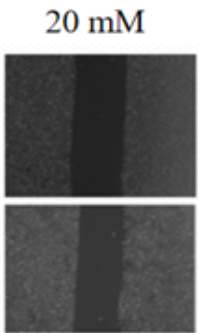

$20 \mathrm{mM}$
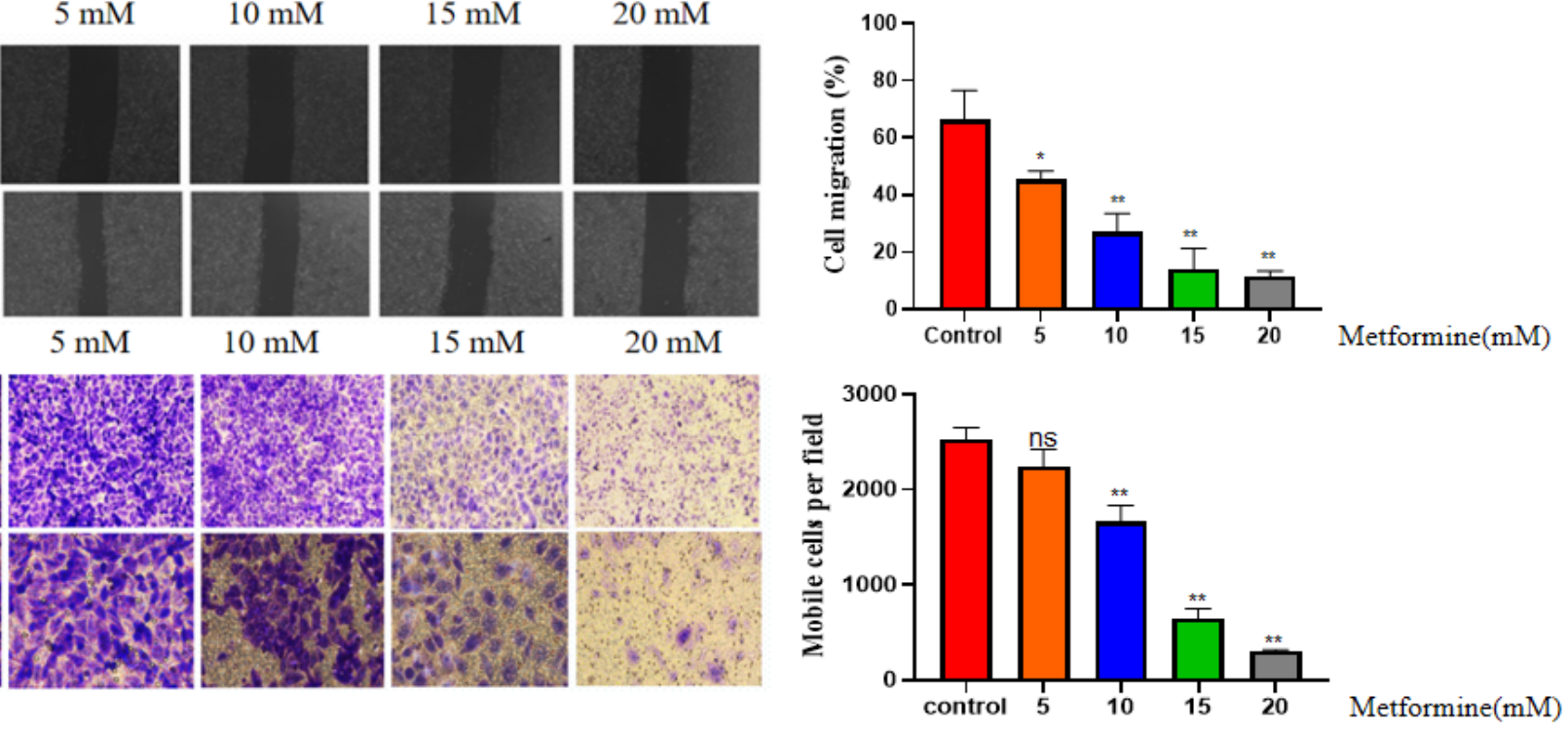

B)5637
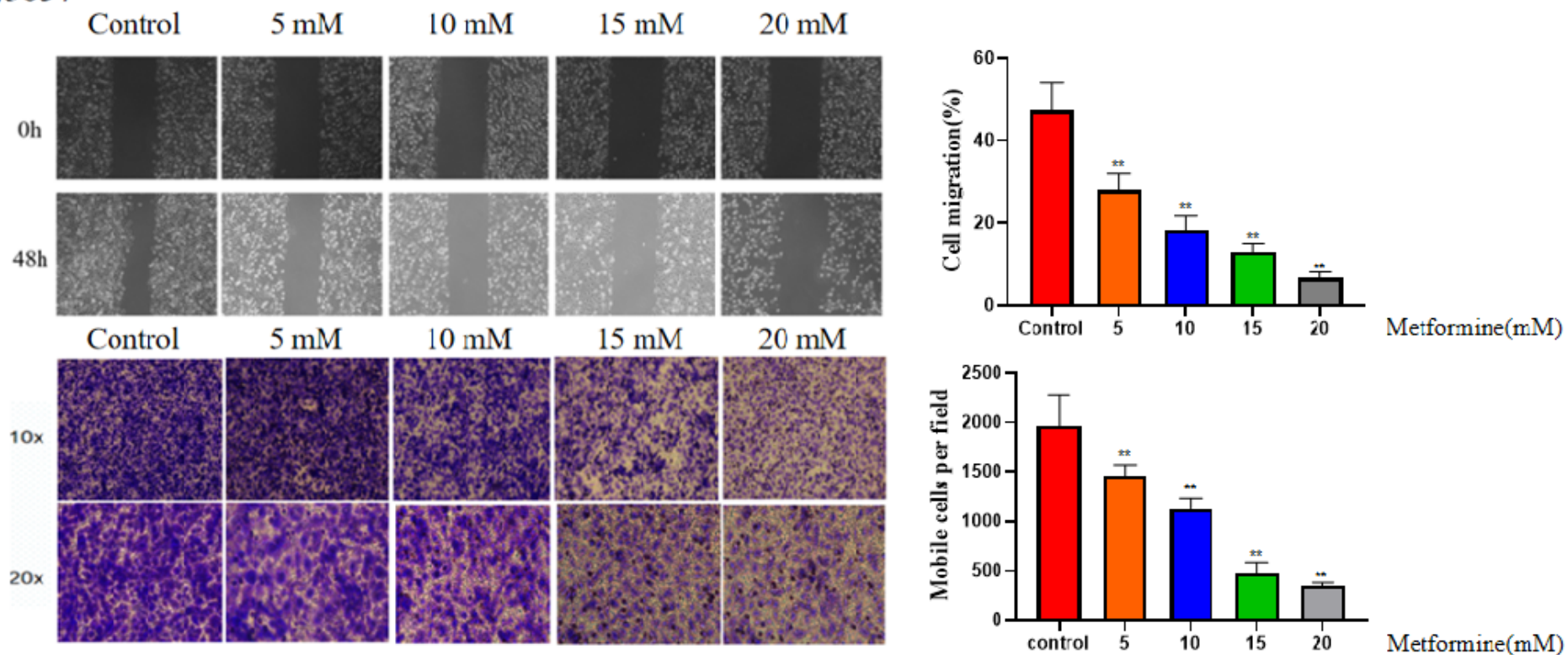

Figure 1

The effect of metformin on the migration of T24 and 5637 cell lines. The effect of different concentrations of metformin on the migration ability of T24 (A) and 5637 (B) cell lines was observed for 0-48 hours (magnification, 10-20x). The results are shown as the mean \pm the standard deviation (SD) from three independent experiments and were compared with the values for the blank control $\left({ }^{\star} P<0.05\right.$, $\star * P<0.01)$. 

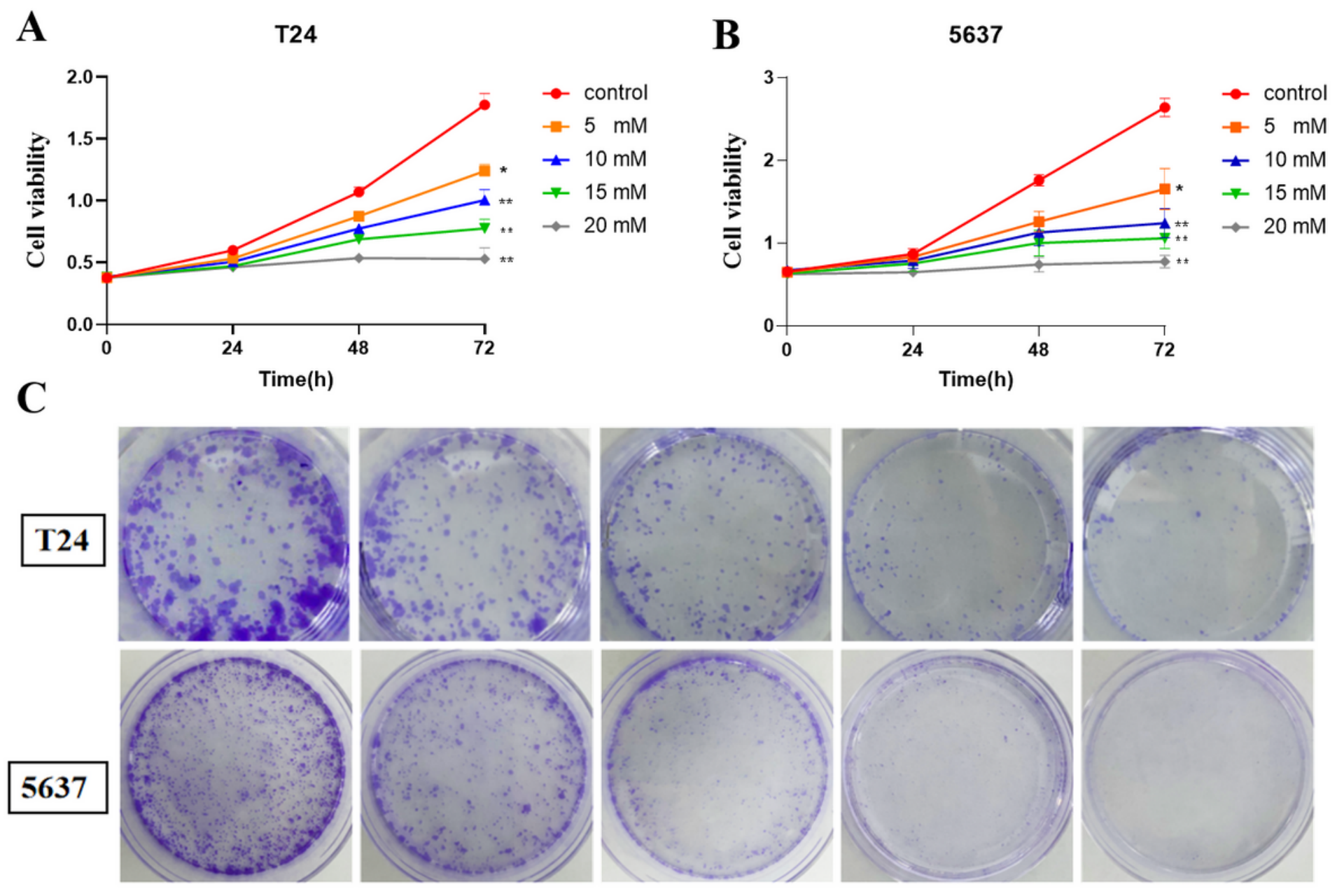

Figure 2

The effects of metformin at different concentrations on the proliferation of T24 and 5637 cell lines. A, The effects of different concentrations of metformin over time on the proliferative ability of T24 cell lines were observed using CCK-8 assays to measure proliferation. B, The effects of different concentrations of metformin over time on the proliferative ability of 5637 cell lines were observed using CCK-8 assays to measure cell viability. C, The effects of metformin at different concentrations on the proliferation of T24 cell lines assessed using colony formation experiments. The results are shown as the mean \pm the standard deviation (SD) from three independent experiments and were compared with the values for the control $\left({ }^{*} P<0.05,{ }^{*} \mathrm{P}<0.01\right)$. CCK -8 , cell counting kit 8 . 
A) $\mathbf{T 2 4}$
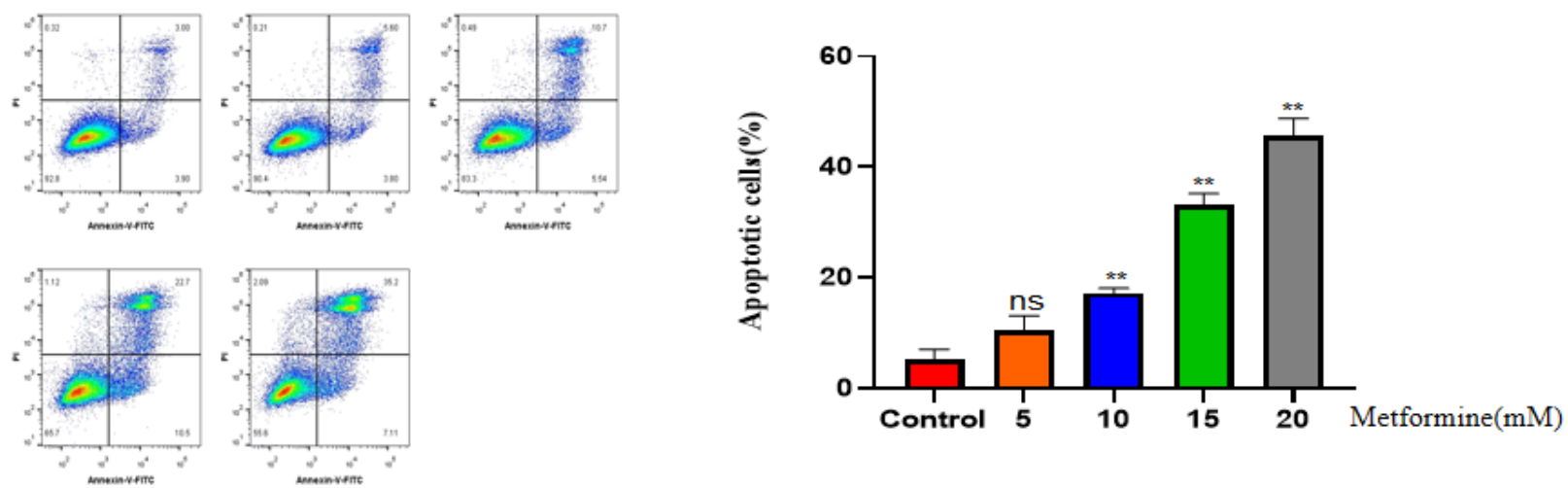

B

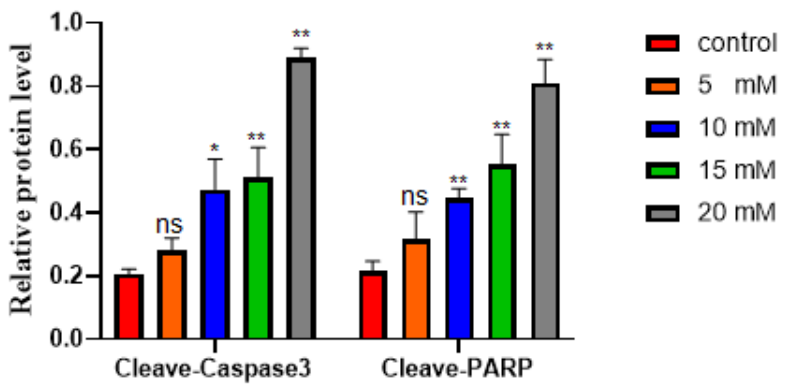

\section{C) 5637}
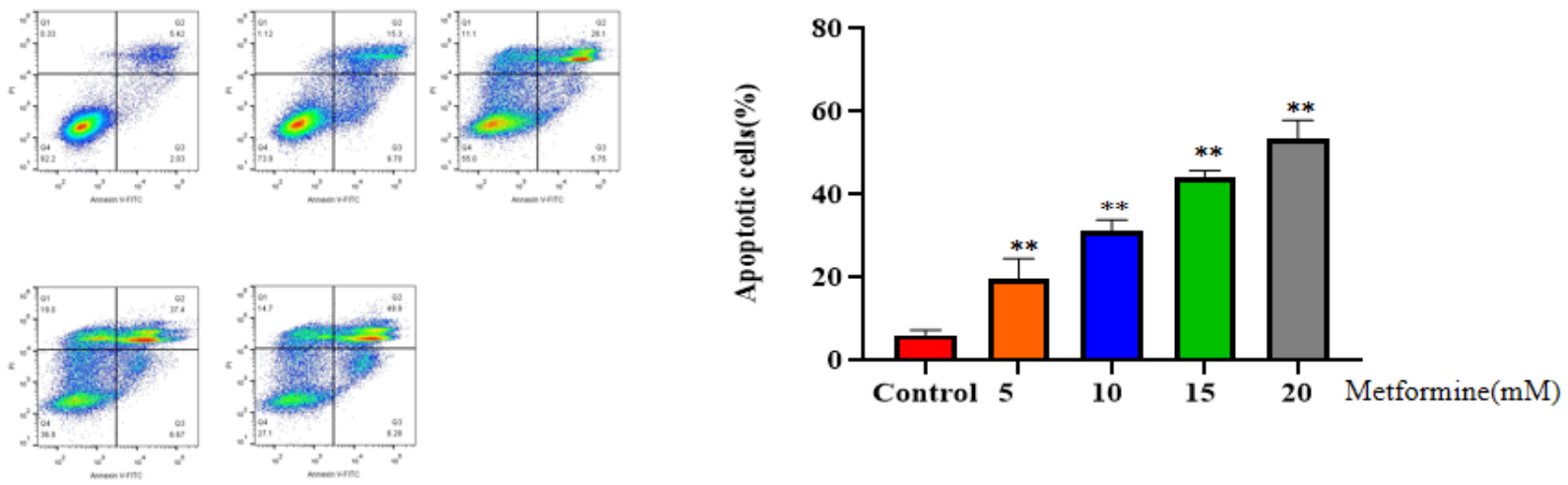

D
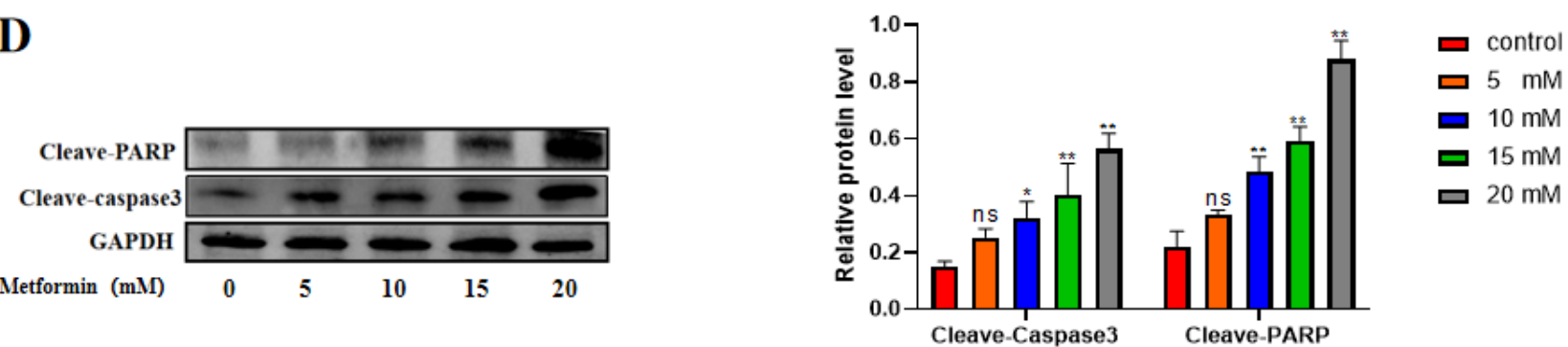

\section{Figure 3}

In vitro, cell apoptosis was promoted by different of concentrations of metformin. A, T24 cell apoptosis as detected using flow cytometry. The images show the number of Q2 + Q4 as apoptotic cells. B, Western blotting detection of the levels of cleaved-caspase 3 and cleave-PARP in T24 cells. C, 5637 cell apoptosis as detected using flow cytometry. The images show the number of Q2 + Q4 as apoptotic cells. D, Western blotting detection of the levels of cleaved-caspase 3 and cleave-PARP in 5637 cells. The loading control 
comprised GAPDH. The representative column diagrams showing results of relative protein expression. In the graphs, the results are shown as the mean \pm the standard deviation (SD) from three independent experiments and were compared with the values for the control ( $\left.{ }^{*} \mathrm{P}<0.05,{ }^{\star *} \mathrm{P}<0.01\right)$. PARP, poly (ADPribose) polymerase GAPDH, glyceraldehyde-3-phosphate dehydrogenase.

\section{A)T24}
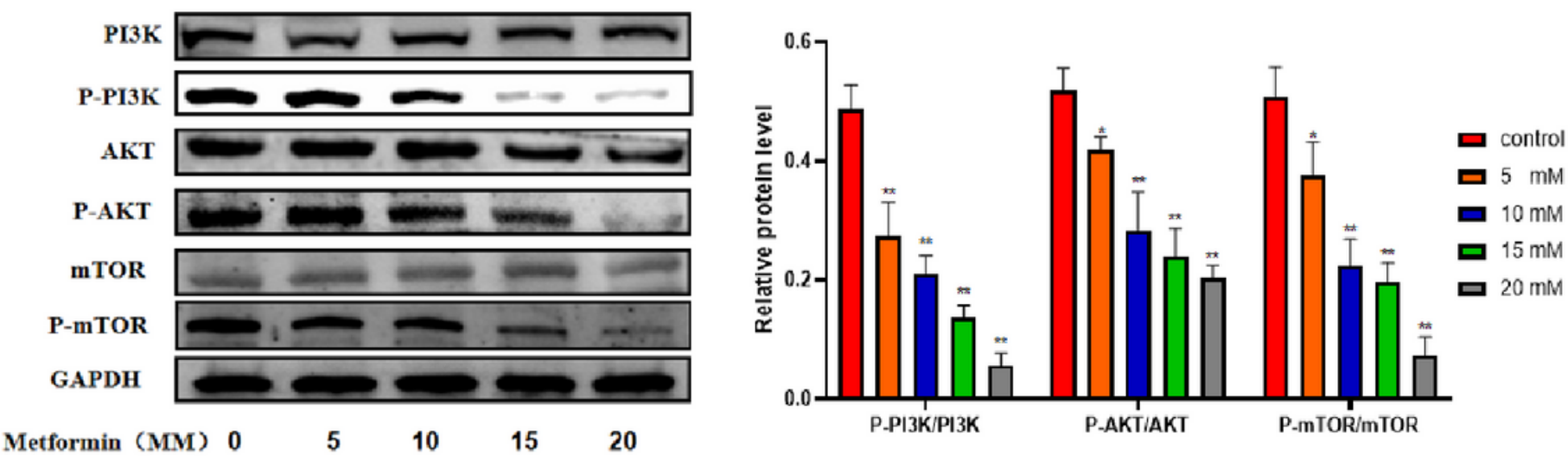

B)5637
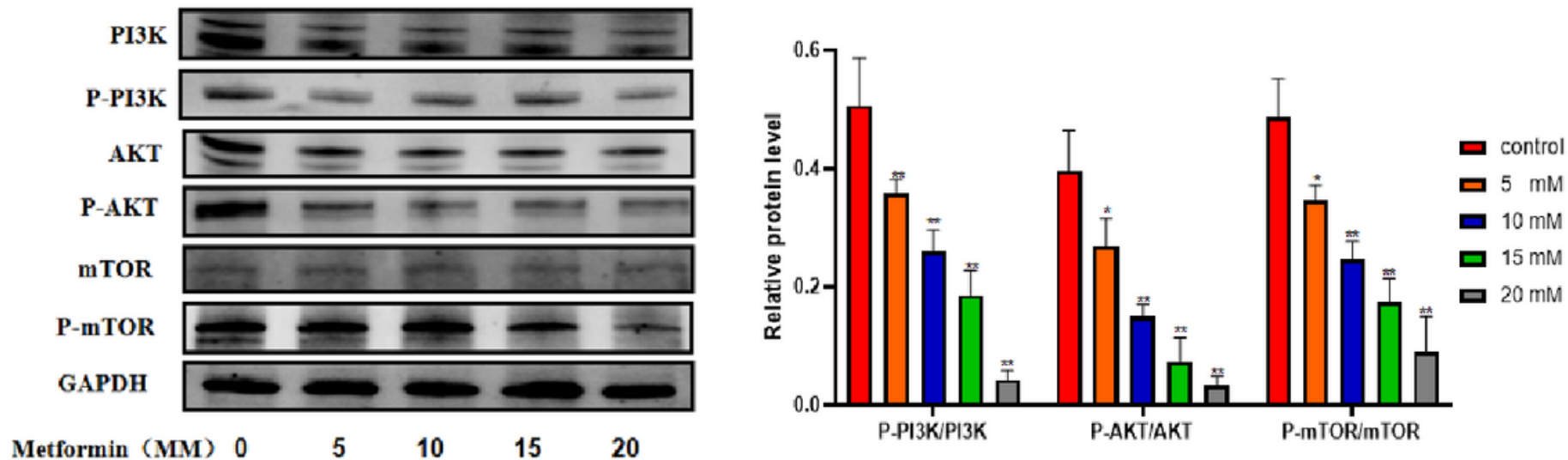

Figure 4

The different of concentrations of Metformin inhibited PI3K/AKT/mTOR pathway activation in vitro. A, Western blotting detection of PI3K/AKT/mTOR and p-PI3K/p-AKT/p-mTOR protein levels in T24 cells. The loading control comprised GAPDH. The graph shows the relative protein levels. B, Western blotting detection of PI3K/AKT/mTOR and p-PI3K/p-AKT/p-mTOR protein levels in 5637 cells. The loading control comprised GAPDH. The graph shows the relative protein levels. Data are shown as the mean \pm SD of three independently performed experiments ( ${ }^{*} \mathrm{P}<0.05$, $\left.{ }^{*} \mathrm{P}<0.01\right)$. PI3K, phosphatidylinositol-4,5bisphosphate 3-kinase; p-PI3K, phosphorylated PI3K; Akt, protein kinase B; p-Akt, phosphorylated Akt; mTOR, mechanistic target of rapamycin; p-mTOR, phosphorylated mTOR; GAPDH, glyceraldehyde-3phosphate dehydrogenase.

\section{Supplementary Files}


This is a list of supplementary files associated with this preprint. Click to download.

- 5637WesternblotsBrands.zip

- T24WesternblotsBrands.zip 